\title{
Zero-in-the-spectrum conjecture on regular covers of compact manifolds
}

Piotr W. Nowak

\begin{abstract}
We prove the zero-in-the-spectrum conjecture for large, regular covers associated to amenable subgroups of fundamental group of a closed manifold $\mathcal{N}$, provided that $\pi_{1}(\mathcal{N})$ is $C^{*}$-exact.
\end{abstract}

Mathematics Subject Classification (2000). Primary 58J22; Secondary 58J50.

Keywords. Property A, exact group, zero-in-the-spectrum conjecture.

The zero-in-the-spectrum conjecture was first formulated by Gromov [6], [7] and asks if the spectrum Laplace-Beltrami operator acting on the square-integrable $p$-forms on the universal cover of a closed aspherical manifold contains zero. This fact is implied by the Strong Novikov Conjecture and thus the interest in finding a counterexample. A more general zero-in-the-spectrum conjecture on open complete manifolds was stated by Lott and it is true if there is a positive answer to the following question: does the spectrum of the Laplace-Beltrami operator $\Delta_{p}$ acting on square-integrable $p$-forms of a complete manifold $\mathcal{M}$ contain zero for some $p=0,1, \ldots$ ? The answer is negative in general: Farber and Weinberger [4] showed that for every $n \geq 6$ there exists a manifold $\mathcal{N}$ such that zero is not in the spectrum of $\Delta_{p}$ for any $p \in 0,1, \ldots$ acting on the universal cover of $\mathcal{N}$. Later Higson, Roe and Schick [12] extended this result and gave a complete description of groups which can appear as fundamental groups of manifolds whose universal covers do not have zero in the spectrum of the Laplacian.

Because of the origins of the problem, various covering spaces are a natural environment for considering zero-in-the-spectrum questions. An early result of this type is a theorem of Brooks [1] stating that given a regular cover $\mathcal{M}$ of a compact manifold $\mathcal{N}, 0$ is in the spectrum of $\Delta_{0}$ on $\mathcal{M}$ if and only if the group of deck transformations is amenable. The articles [14], [15] provide a comprehensive survey of this topic.

The purpose of this article is to prove the zero-in-the-spectrum conjecture on certain regular covers associated to normal, amenable subgroups of the fundamental 
group of a compact manifold $\mathcal{N}$ (we call such covers co-amenable) under the assumption that $\pi_{1}(\mathcal{N})$ is $C^{*}$-exact, or in other words, has Yu's Property A. This result (Theorem 7) is stated and proved in the last section, since to formulate it we need a few technical notions, which are explained in detail in the text.

Our main tool is coarse index theory. In order to apply such methods we need to consider manifolds satisfying a certain largeness condition, which allows to translate small scale geometric information to large scale. More precisely we call an open $n$-manifold $\mathcal{M}$ large if the fundamental class in the locally finite homology group $H_{n}^{\text {lf }}(\mathcal{M})$ survives the coarsening of this homology, giving a non-zero element in the coarse homology group $H X_{n}(\mathcal{M})$ (see Section 2 for a precise formulation). Roughly speaking this means that the image of the fundamental class of the manifold $\mathcal{M}$ under the classifying map to (appropriately constructed) locally finite homology of the classifying space $E G$, where $G$ is the group of deck transformations of $\mathcal{M}$, is nonzero. This notion is essential for us since the examples of manifolds we are concerned with, as regular covers associated to non-trivial subgroups of the fundamental groups of compact manifolds, do not satisfy another widely used largeness condition, uniform contractibility.

The paper is organized as follows. In Section 1 we discuss $C^{*}$-exactness and prove a permanence property for exact groups, in Section 2 we define in detail the above-mentioned notion of largeness and the last section is devoted solely to proving the main theorem of the paper.

\section{1. $C^{*}$-exact quotients}

Property A was defined by Yu [22] as a geometric condition implying the Coarse Baum-Connes Conjecture for discrete metric spaces. Soon after it was introduced it turned out that Property A for finitely generated group $G$ is equivalent to existence of a topologically amenable action on a compact space [11] as well as to exactness of the reduced group $C^{*}$-algebra $C_{r}^{*}(G)$ [9], [18]. The latter is the reason why groups with Property A are often called $C^{*}$-exact, or simply exact, and we will use these terms interchangeably.

We denote

$$
\ell_{1}(X)_{1,+}=\left\{f \in \ell_{1}(X) \mid\|f\|_{1}=1, f \geq 0\right\} .
$$

Definition 1 ([22]; in this form [11]). Let $X$ be a discrete metric space with bounded geometry. We say that $X$ has Property A if for every $R<\infty$ and $\varepsilon>0$ there exists a map $\xi: X \rightarrow \ell_{1}(X)_{1,+}$ and $S \in \mathbb{R}$ such that

(1) $\left\|\xi_{x}-\xi_{y}\right\|_{\ell_{1}(X)} \leq \varepsilon$ whenever $d(x, y) \leq R$,

(2) $\operatorname{supp} \xi_{x} \subseteq B(x, S)$ for every $x \in X$. 
Determining how large the class of groups possessing Property A is an active area of research due to applications to various problems in geometry and topology, see for example [19], [20], [21], [22], [23]. One of the most natural topics in this context are permanence properties of exact groups. Such properties were studied most notably by Kirchberg and Wasserman [13] using the $C^{*}$-algebraic definition and Dadarlat and Guentner in the context of coarse embeddability into the Hilbert space [3]. Among such constructions are passing to a subgroup, direct limits and extensions thus in many ways Property A indeed resembles amenability.

Unlike amenability however, Property A is preserved by free products [2] and, more important, is not preserved under surjective homomorphisms. The latter is a consequence of Gromov's construction [8] of finitely presented groups which do not coarsely embed into the Hilbert space together with exactness of free groups. Thus we are interested in general conditions guaranteeing that in the exact sequence

$$
1 \longrightarrow H \longrightarrow G \longrightarrow G / H \longrightarrow 1
$$

$G / H$ has Property A provided that $G$ has it. If we exclude the trivial case and assume that $G / H$ is infinite there are two other cases in which such a statement is obviously true:

(i) If $H$ is a finite group then $G$ and $G / H$ are quasi-isometric, so $G$ is exact if and only if $G / H$ is exact.

(ii) If the exact sequence (1) splits then $G / H$ is embedded in $G$, and inherits exactness from the ambient group.

Our first step in proving Theorem 7 is the following

Theorem 2. Let $G$ be a finitely generated group satisfying Property $A$ and let $H$ be an amenable subgroup of $G$. Then the quotient $G / H$ has Property $A$.

This condition is sharp: as soon as we drop the amenability condition on $H$, we can take $G$ in (1) to be a free group and get all finitely generated groups as quotients.

Let $G$ be a finitely generated group and $H$ its subgroup. Then the action of $H$ on $G$ by translations induces an isometric action of $H$ on $\ell_{\infty}(G)$. We denote this action by $(h \cdot f)(x)=f\left(h^{-1} x\right)$, for every $f \in \ell_{\infty}(G), x \in G$ and $h \in H$. If $G$ is amenable then given $f \in \ell_{\infty}(G)$ we will denote the value of the invariant mean of $f$ by

$$
\int_{G} f(g) d g
$$

We will treat the cosets of $H$ as orbits of its action on $G$ and denote the orbit of $x \in G$ by $H x$. The quotient $G / H$ is a metric space with the metric

$$
d(H x, H y)=\min _{h, h^{\prime} \in H} d\left(h x, h^{\prime} y\right) .
$$


This in particular means that the quotient map $G \rightarrow G / H$ is a contraction.

In [16], [17] an averaging procedure for Property A was obtained and the next statement also makes use of it.

Proposition 3. Let $G$ be a group with Property $A$ and let $H$ be an amenable subgroup of $G$. Then for every $\varepsilon>0$ Property $A$ can be realized by a map $\xi: G \rightarrow \ell_{1}(G)_{1,+}$ such that $\xi$ is equivariant under the action of $H$, i.e.,

$$
\xi_{h x}=h \cdot \xi_{x}
$$

for every $h \in H$ and $x \in G$.

Proof. Assume that $G$ satisfies conditions of Definition 1 for $R<\infty, \varepsilon>0$ with $S>0$ realized by a function $\zeta: G \rightarrow \ell_{1}(G)_{1,+}$. For every $x \in G$ define

$$
\xi_{x}(y)=\int_{H} \zeta_{h x}(h y) d h .
$$

Since $0 \leq \zeta_{x}(y) \leq 1$ for all $x, y \in G$ we get a well-defined function $\xi_{x}: G \rightarrow \mathbb{R}$ satisfying $0 \leq \xi_{x}(y) \leq 1$ for all $x, y \in G$. Observe that if $d(x, y)>S$ then $\zeta_{x}(y)=0$. Since $H$ acts by isometries, $d(h x, h y)=d(x, y)$ and it follows that $\xi_{x}(y)=0$ if $d(x, y)>S$. This allows to compute the norm of $\xi_{x}$ :

$$
\begin{aligned}
\left\|\xi_{x}\right\|_{\ell_{1}(G)} & =\sum_{y \in B(x, S)} \xi_{x}(y)=\sum_{y \in B(x, S)} \int_{H} \zeta_{h x}(h y) d h \\
& =\int_{H}\left(\sum_{y \in B(x, S)} \zeta_{h x}(h y)\right) d h=\int_{H} 1 d h \\
& =1
\end{aligned}
$$

which shows that $\xi_{x}$ is an element of $\ell_{1}(G)_{1,+}$ for every $x \in G$.

Let now $x_{1}, x_{2} \in G$ satisfy $d\left(x_{1}, x_{2}\right)=1$. Then

$$
\begin{aligned}
\left\|\xi_{x_{1}}-\xi_{x_{2}}\right\|_{\ell_{1}(G)} & =\sum_{y \in G}\left|\xi_{x_{1}}(y)-\xi_{x_{2}}(y)\right| \\
& =\sum_{y \in G}\left|\int_{H} \zeta_{h x_{1}}(h y) d h-\int_{H} \zeta_{h x_{2}}(h y) d h\right| \\
& \leq \int_{H}\left(\sum_{y \in X}\left|\zeta_{h x_{1}}(h y)-\zeta_{h x_{2}}(h y)\right|\right) d h \\
& \leq \int_{H} \varepsilon d h=\varepsilon
\end{aligned}
$$


since the sum is in fact finite and the metric is left-invariant.

Finally we need to show that (2) holds. Indeed, if $\gamma \in H$ and $x \in G$ then by the invariance of the mean on $H$ we obtain

$$
\begin{aligned}
\xi_{\gamma x}(y) & =\int_{H} \zeta_{h \gamma x}(h y) d h \\
& =\int_{H} \zeta_{\tilde{h} x}\left(\tilde{h} \gamma^{-1} y\right) d \tilde{h}=\xi_{x}\left(\gamma^{-1} y\right) \\
& =\gamma \cdot \xi_{x}(y),
\end{aligned}
$$

after substituting $\tilde{h}=h \gamma$. This ends the proof.

Proof of Theorem 2. By Proposition 3, the function $\xi: G \rightarrow \ell_{1}(G)$ can be chosen to be equivariant on cosets of $H$. We define the map $\eta: G / H \rightarrow \ell_{1}(G / H)_{1,+}$ by

$$
\eta_{H x}(H y)=\sum_{h \in H} \xi_{x}(h y) .
$$

We need to show that $\eta$ is well defined. So let $H x=H x^{\prime}$ and $H y=H y^{\prime}$ as elements of $G / H$. Then there are elements $\gamma, g \in H$ such that $\gamma x=x^{\prime}$ and $g y=y^{\prime}$.

$$
\begin{aligned}
\eta_{H x^{\prime}}\left(H y^{\prime}\right) & =\sum_{h \in H} \xi_{x^{\prime}}\left(h y^{\prime}\right)=\sum_{h \in H} \xi_{\gamma x}(h g y) \\
& =\sum_{h \in H} \xi_{x}\left(\gamma^{-1} h g y\right)=\sum_{h \in H} \xi_{x}(h y) \\
& =\eta_{H x}(H y),
\end{aligned}
$$

after using Lemma 3 and substituting $\gamma^{-1} h g$ for $g$.

Now note that since the quotient map $G \rightarrow G / H$ is a contraction, if the elements $H x$ and $H y$ are more than distance $S$ away from each other, $\xi_{x}$ vanishes on the coset $H y$ and we have

$$
\eta_{H x}(H y)=\sum_{h \in H} \xi_{x}(h y)=0
$$

thus supp $\eta_{H x} \subseteq B(H x, S)$ and we also have

$$
\begin{aligned}
\left\|\eta_{H x}\right\|_{\ell_{1}(G / H)} & =\sum_{H y \in G / H} \eta_{H x}(H y) \\
& =\sum_{H y \in G / H} \sum_{h \in H} \xi_{x}(h y) \\
& =\left\|\xi_{h}\right\|_{\ell_{1}(G / H)}=1 .
\end{aligned}
$$


Suppose now that elements $H x$ and $H x^{\prime}$ are of distance 1 from each other. This means that the elements $x$ and $x^{\prime}$ can be assumed to satisfy $d\left(x, x^{\prime}\right) \leq 1$. Thus

$$
\begin{aligned}
\left\|\eta_{H x}-\eta_{H x^{\prime}}\right\|_{\ell_{1}(G / H)} & =\sum_{H y \in G / H}\left|\eta_{H x}(H y)-\eta_{H x^{\prime}}(H y)\right| \\
& =\sum_{H y \in G / H}\left|\sum_{h \in H} \xi_{x}(h y)-\sum_{h \in H} \xi_{x^{\prime}}(h y)\right| \\
& \leq \sum_{H y \in G / H} \sum_{h \in H}\left|\xi_{x}(h y)-\xi_{x^{\prime}}(h y)\right| \\
& =\left\|\xi_{x}-\xi_{x^{\prime}}\right\|_{\ell_{1}(G)} \leq \varepsilon .
\end{aligned}
$$

Remark 4. Note that taking quotients by amenable subgroups not only preserves Property A, but also preserves a coarse invariant associated to an exact group. In [17] an invariant $\mathrm{A}_{X}$ for metric spaces with Property A was defined and one can extract from the proof of Theorem 2 that for an amenable subgroup $H$ of a group $G$ satisfying Property A we have

$$
\mathrm{A}_{G / H} \leq \mathrm{A}_{G}
$$

We end this section with a question. It is a very interesting and so far unsolved problem whether Thompson's group $F$ has Property A. One strategy to obtain a positive answer could use Theorem 2 and it becomes natural to ask: is it possible to write Thompson's group $F$ in the form $G / H$, where $G$ has Property $A$ and $H$ is amenable? It would be sufficient to embed $F$ into such $G / H$ coarsely, e.g. as a subgroup.

\section{Large Riemannian manifolds}

2.1. Largeness. There are several notions of largeness of open manifolds, see e.g. [6]. One of them is uniform contractibility, which means that for every $R<\infty$ there exists an $S_{R}<\infty$ such that for any point $x \in \mathcal{M}$ the ball $B(x, R)$ is contractible inside $B\left(x, S_{R}\right)$. We will need a less restrictive criterion.

Let $X$ be a metric space. An anti-Čech system is a sequence $\left\{U_{k}\right\}_{k \in \mathbb{N}}$ of covers such that:

(i) there exist numbers $R_{k}, k=1,2, \ldots$, such that $\operatorname{diam}(U) \leq R_{k}$ for every $U \in U_{k}$

(ii) the Lebesque numbers $\lambda_{k}$ of $U_{k}$ satisfy $\lambda_{k} \geq R_{k-1}$;

(iii) $\lambda_{k} \rightarrow \infty$ as $k \rightarrow \infty$. 
Let $H_{*}^{\text {lf }}$ be the locally finite homology theory. The coarse homology $H X_{*}(X)$ [19] is defined by setting

$$
H X_{*}(X)=\lim _{k \rightarrow \infty} H_{*}^{\mathrm{lf}}\left(\left|U_{k}\right|\right)
$$

where $\left\{U_{k}\right\}$ is an anti-Čech system for $X$ and $\left|U_{k}\right|$ denotes the nerve space of the cover $U_{k}$; see also [10]. There is a character map $c_{*}: H_{*}^{\text {lf }}(X) \rightarrow H X_{*}(X)$ induced by the map $c: X \rightarrow\left|U_{1}\right|$ defined by the formula

$$
c(x)=\sum_{U \in U_{1}} \varphi_{U}(x)[U]
$$

where $\left\{\varphi_{U}\right\}_{U_{1}}$ is a partition of unity subordinate to the cover $U_{1}$. The character map $c_{*}$ is an isomorphism provided that $X$ is uniformly contractible [19], see also [10].

Definition 5 ([5]). Let $\mathcal{M}$ be a complete, oriented $n$-dimensional manifold. Let $[\mathcal{M}] \in H_{n}^{\mathrm{lf}}(\mathcal{M})$ be the fundamental class of $\mathcal{M}$. We call $\mathcal{M}$ large if

$$
c_{*}([\mathcal{M}]) \neq 0 .
$$

In [5] the above condition was called macroscopical largeness. Note that the above notion of largeness for equivalent metrics depends only on the quasi-isometry class of these metrics. More precisely, take a manifold $\mathcal{M}$ which is equipped with two equivalent, quasi-isometric metrics, $d_{1}$ and $d_{2}$ and the corresponding character maps by $c_{*}^{1}$ and $c_{*}^{2}$ respectively. Then for any $n$ the diagram

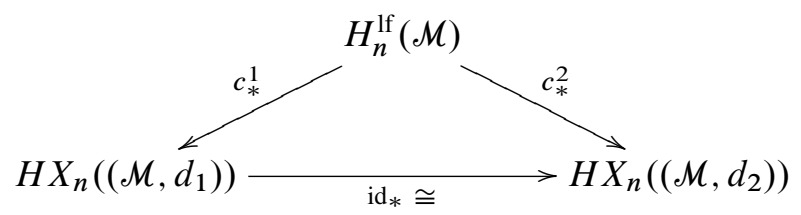

is commutative, so in particular $\left(\mathcal{M}, d_{1}\right)$ is large if and only if $\left(\mathcal{M}, d_{2}\right)$ is.

Remark 6. Note also that largeness is the same as asking whether $e_{*}([\mathcal{M}]) \neq 0$ where $e: \mathcal{M} \rightarrow E G$ is the classifying map of the cover $\mathcal{M}$ and $e_{*}$ is the map induced on appropriately constructed homology. One has to be careful and define this homology as an appropriate direct limit over compact subsets, as $E G$ might not be locally compact. We skip the details. 


\section{The main theorem}

Let $\left(\mathcal{M}, d_{\mathcal{M}}\right)$ be an open complete Riemannian manifold and let $G$ be a group acting freely, properly on $\mathcal{M}$ by isometries with a compact quotient $\mathcal{N}=\mathcal{M} / G$. We have the following exact sequence:

$$
1 \longrightarrow \pi_{1}(\mathcal{M}) \longrightarrow \pi_{1}(\mathcal{N}) \longrightarrow G \longrightarrow 1
$$

In the above setting we will say that $\mathcal{M}$ is a co-amenable cover if $\pi_{1}(\mathcal{M})$ is amenable ( $\mathcal{M}$ is often called an amenable cover when $G$ is amenable). Obviously if $\pi_{1}(\mathcal{M})$ is non-trivial, the manifold $\mathcal{M}$ is not uniformly contractible.

Theorem 7. Let $\left(\mathcal{N}, d_{\mathcal{N}}\right)$ be a closed Riemannian manifold such that $\pi_{1}(\mathcal{N})$ is $C^{*}$ exact. Let $\left(\mathcal{M}, d_{\mathcal{M}}\right)$ be a co-amenable cover of $\mathcal{N}$ which is large and has bounded geometry (i.e. bounded sectional curvature and positive injectivity radius). Then the zero-in-the-spectrum conjecture holds for $\mathcal{M}$ with any bounded geometry metric which is quasi-isometric and topologically equivalent to $d_{\mathcal{M}}$.

Proof of Theorem 7. Let $\mathcal{M}, \mathcal{N}$ and $G$ be as above. By assumptions and Theorem 2 we have that $G$ has Property A. By the Švarc-Milnor lemma, $G$ and $\mathcal{M}$ are quasiisometric due to the fact that $\mathcal{N}=\mathcal{M} / G$ is compact. Since $G$ has Property A and Property $\mathrm{A}$ is a coarse invariant, $\mathcal{M}$ equipped with any metric in the quasi-isometry class of $d_{\mathcal{M}}$ has Property A.

The index map is defined as the following composition:

$$
K_{*}(\mathcal{M}) \stackrel{c_{*}}{\longrightarrow} K X_{*}(\mathcal{M}) \stackrel{\mu}{\longrightarrow} K_{*}\left(C^{*}(\mathcal{M})\right)
$$

where $K_{*}(\mathcal{M})$ is the $K$-homology group of $\mathcal{M}, K X_{*}(\mathcal{M})$ is the coarsening of this $K$-homology group, $C^{*}(\mathcal{M})$ is the Roe algebra, $\mu$ is the coarse assembly map and $c_{*}$ is the character map (see [10], [19], [20], [22] for details on the Coarse Baum-Connes Conjecture).

Let $\mathscr{D}$ be the de Rham operator on $\mathcal{M}$. Since $\mathcal{M}$ is large, we have that $c_{*}(\mathscr{D}) \neq 0$ in the coarse $K$-homology group $K X_{*}(\mathcal{M})$. By Theorems 2.2 and 1.1 in [22], Property A for $\mathcal{M}$ implies that the Coarse Baum-Connes Conjecture is true for $\mathcal{M}$, i.e. $\mu$ is an isomorphism and consequently $\mu \circ c_{*}(\mathscr{D}) \neq 0$ in $K_{*}\left(C^{*}(\mathcal{M})\right)$. This ends the proof.

In the case $\pi_{1}(\mathcal{M})=\{1\}$ (i.e. the cover is universal), Theorem 7 is due to Yu [22].

The assumption of largeness cannot be dropped as the example of Farber and Weinberger [4] shows (see also [12]), since in their construction the fundamental group of the manifold $\mathcal{N}$ is a direct product of free groups, which is exact. 
Vol. 84 (2009) Zero-in-the-spectrum conjecture on regular covers of compact manifolds

\section{References}

[1] R. Brooks, The fundamental group and the Spectrum of the Laplacian. Comment. Math. Helv. 56 (4) (1981), 581-598. Zbl 0495.5802 MR 0656213

[2] X. Chen, M. Dadarlat, E. Guentner, G. Yu, Uniform embeddability and exactness of free products. J. Funct. Anal. 205 (1) (2003), 168-179. Zbl 1041.46043 MR 2020212

[3] M. Dadarlat, E. Guentner, Constructions preserving Hilbert space uniform embeddability of discrete groups. Trans. Amer. Math. Soc. 8 (2003), 3253-3275. Zbl 1028.46104 MR 1974686

[4] M. Farber, S. Weinberger, On the zero-in-the-spectrum conjecture. Ann. of Math. (2) 154 (1) (2001), 139-154. Zbl 0992.58012 MR 1847591

[5] G. Gong, G. Yu, Volume growth and positive scalar curvature. Geom. Funct. Anal. 10 (4) (2000), 821-828. Zbl 0979.53033 MR 1791141

[6] M. Gromov, Large Riemannian manifolds. In Curvature and topology of Riemannian manifolds (Katata, 1985), Lecture Notes in Math. 1201, Springer-Verlag, Berlin 1986, 108-121. Zbl 0601.53038 MR 0859578

[7] M. Gromov, Asymptotic invariants of infinite groups. London Math. Soc. Lecture Notes Ser. 182, Cambridge University Press, Cambridge 1993. Zbl 0841.20039 MR 1253544

[8] M. Gromov, Random walk in random groups. Geom. Funct. Anal. 13 (1) (2003), 73-146. Zbl 1122.20021 MR 1978492

[9] E. Guentner, J. Kaminker, Exactness and the Novikov conjecture. Topology 41 (2) (2002), 411-418. Zbl 0992.58002 MR 1876896

[10] N. Higson, J. Roe, On the coarse Baum-Connes conjecture. In Novikov conjectures, index theorems and rigidity (Oberwolfach, 1993), Vol. 2, London Math. Soc. Lecture Note Ser. 227, Cambridge University Press, Cambridge 1995, 227-254. Zbl 0957.58019 MR 1388312

[11] N. Higson, J. Roe, Amenable group actions and the Novikov conjecture. J. Reine Angew. Math. 519 (2000), 143-153. Zbl 0964.55015 MR 1739727

[12] N. Higson, J. Roe, T. Schick, Spaces with vanishing $l^{2}$-homology and their fundamental groups (after Farber and Weinberger). Geom. Dedicata 87 (1-3) (2001), 335-343. Zbl 0991.57002 MR 1866855

[13] E. Kirchberg, S. Wassermann, Permanence properties of $C^{*}$-exact groups. Doc. Math. 4 (1999), 513-558. Zbl 0958.46036 MR 1725812

[14] J. Lott, The zero-in-the-spectrum question. Enseign. Math. (2) 42 (3-4) (1996), 341-376. Zbl 0874.58086 MR 1426443

[15] W. Lück, $L^{2}$-invariants of regular coverings of compact manifolds and CW-complexes. In Handbook of geometric topology, North-Holland, Amsterdam 2002, 735-817. Zbl 1069.57017 MR 1886681

[16] P. W. Nowak, Coarsely embeddable metric spaces without Property A. J. Funct. Anal. 252 (1) (2007), 126-136. Zbl 1142.46038 MR 2357352

[17] P. W. Nowak, On exactness and isoperimetric profiles of discrete groups. J. Funct. Anal. 243 (1) (2007), 323-344. Zbl 1117.60008 MR 2291440 
[18] N. Ozawa, Amenable actions and exactness for discrete groups. C. R. Acad. Sci. Paris Ser. I Math. 330 (2000), 691-695 Zbl 0953.43001 MR 1763912

[19] J. Roe, Coarse cohomology and index theory on complete Riemannian manifolds. Mem. Amer. Math. Soc. 104 (497) (1993). Zbl 0780.58043 MR 1147350

[20] J. Roe, Index theory, coarse geometry, and topology of manifolds. CBMS Regional Conf. Ser. in Math. 90, Amer. Math. Soc., Providence, RI, 1996. Zbl 0853.58003 MR 1399087

[21] G. Yu, Zero-in-the-spectrum conjecture, positive scalar curvature and asymptotic dimension. Invent. Math. 127 (1) (1997), 99-126. Zbl 0889.58082 MR 1423027

[22] G. Yu, The coarse Baum-Connes conjecture for spaces which admit a uniform embedding into Hilbert space. Invent. Math. 139 (2000), 201-240. Zbl 0956.19004 MR 1728880

[23] G. Yu, Higher index theory of elliptic operators and geometry of groups. In International Congress of Mathematicians (Madrid, 2006), Vol. II, EMS Publishing House, Zürich 2006, 1623-1639, Zbl 1104.46044 MR 2275662

Received April 19, 2007

Piotr W. Nowak, Department of Mathematics Texas A\&M University, College Station, TX 77843, U.S.A.

E-mail: pnowak@math.tamu.edu 